\title{
The 3-D extent of the Io UV footprint on Jupiter
}

\author{
B. Bonfond ${ }^{1}$
}

Received 17 March 2010; revised 27 May 2010; accepted 10 June 2010; published 24 September 2010.

[1] The Io footprint (IFP) is the auroral signature of the electromagnetic interaction between Io and Jupiter's magnetosphere. It consists of several spots followed by an extended tail, which are located close to the feet of the magnetic field lines connecting Io to Jupiter. The size of the main spot is a controversial issue, and previously published values range from $\sim 400$ to $\sim 8000 \mathrm{~km}$. However, this question is crucial to understand the processes at play, since this quantity is expected to reflect the size of the interaction region at Io. The present study provides estimates of the size of the Io footprint on a much larger image sample than before, paying a particular attention to the differentiation of the spots and to their 3-D structure. The length of the Main Alfvén Wing (MAW) spot and the length of the trans-hemispheric electron beam (TEB) spot along the footpath are $\sim 850 \mathrm{~km}$, while their width perpendicular to the footpath is $<200 \mathrm{~km}$. Larger lengths are sometimes observed, but these configurations may be attributed to the overlaps of the different spots. The spot lengths are larger than the projected diameter of Io along unperturbed magnetic field lines, which is consistent with recent simulations. The narrowness of the IFP will need to be carefully accounted for in future studies of its brightness. Additionally, the peak altitudes of the MAW and the TEB spot are as high as 900 and $700 \mathrm{~km}$, respectively, which seem to confirm their different origins.

Citation: Bonfond, B. (2010), The 3-D extent of the Io UV footprint on Jupiter, J. Geophys. Res., 115, A09217, doi:10.1029/2010JA015475.

\section{Introduction}

[2] On Earth, the main sources of magnetospheric plasma are the solar wind and the atmospheric escape. On Jupiter, the principal source is Io's intense volcanism which releases around $0.5 \mathrm{t} / \mathrm{s}$ of plasma in the magnetosphere, while an equivalent quantity is lost in the form of energetic neutral atoms [Dols et al., 2008]. Once ionized, the iogenic particles form a dense plasma torus along Io's orbit before diffusing outward in Jupiter's magnetosphere. Because of the tilt of Jupiter's magnetic dipole axis relative to its rotation axis, the torus plane is also tilted relative to Io's orbital plane. Io rotates around Jupiter at an orbital distance of 5.9 Jovian radii $\left(R_{J}\right)$ in a plane roughly perpendicular to Jupiter's rotation axis in approximately $42 \mathrm{~h}$. At this distance, the magnetospheric plasma is forced to corotate with the planet, at one rotation every $\sim 10 \mathrm{~h}$. The relative motion of Io into the torus strongly affects the plasma stream and the magnetic field. This perturbation propagates along the field lines as Alfvén waves and forms an Alfvén wing in each direction. On their way toward Jupiter, these waves undergo filamentation [Chust et al., 2005; Hess et al., 2010] and nonlinear reflections on density gradients [Jacobsen et al., 2007]. Additionally, Jones and Su [2008] and Hess et al. [2010]

\footnotetext{
${ }^{1}$ Laboratoire de Physique Atmosphérique et Planétaire, Université de Liège, Liège, Belgium.

Copyright 2010 by the American Geophysical Union. 0148-0227/10/2010JA015475
}

recently argued that kinetic and inertial effects are most probably the root cause for the electron acceleration that generates both the auroral Io footprints (IFP) on Jupiter and the electron beams observed by the Galileo spacecraft at Io [Williams and Thorne, 2003]. The multiplicity of the spots forming the footprint was first interpreted as reflections of the Alfvén waves on the density gradients at the torus boundaries [Neubauer, 1980; Gérard et al., 2006]. One of the spots is expected to be related to the main Alfvén wing (MAW) while another is related to the reflected Alfvén wing (RAW). However, Bonfond et al. [2008] found that an additional spot is located alternatively upstream or downstream from the MAW spot, depending on the location of Io in the plasma torus. This behavior suggests that this spot is related to transhemispheric electron beams (TEB), i.e., to electrons accelerated upward in one hemisphere and precipitating into the opposite hemisphere.

[3] The purpose of the present study is to provide accurate estimates of the Io footprint size by simultaneously considering each spot individually and by carefully accounting for the three-dimensional structure of these spots. Indeed various and potentially contradictory numbers can be found in the literature concerning the spatial extent of the Io footprint. The first detection of the Io footprint was accomplished in the infrared wavelength of Connerney et al. [1993]. On these images, the IFP appears as a point source, which sets an upper limit to the spot length around $5^{\circ}$. They suggested that the observed extension could be longer than the instantaneous charged particles precipitation area because the lifetime of $\mathrm{H}_{3}^{+}$ 


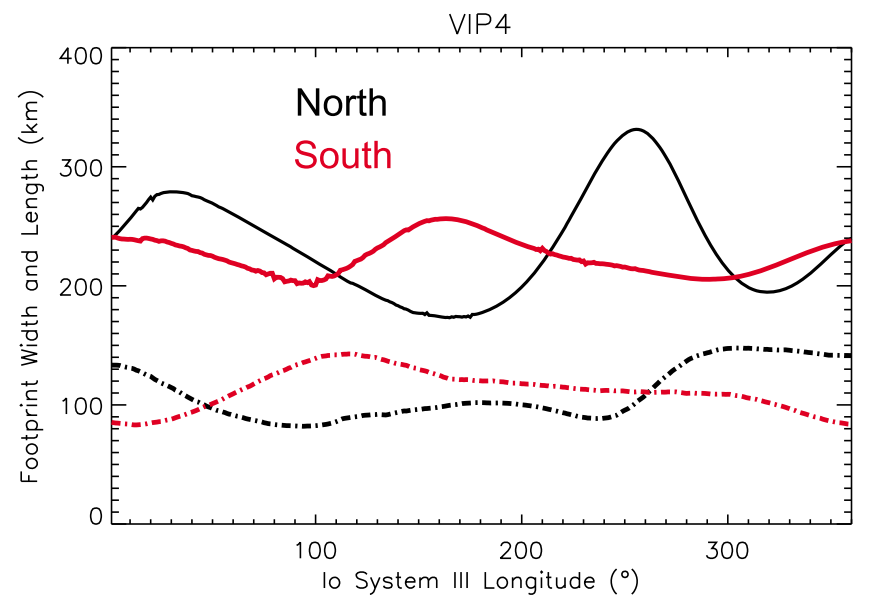

Figure 1. Projected size of Io along unperturbed magnetic field lines as modeled by VIP4. The solid line represents the Io diameter along the Io orbit projected in the Jovian northern and southern ionospheres. This quantity thus stands for the length of the Io flux tube footprint. The dashed line represents the diameter of Io along the Io-Jupiter line projected in the northern and southern Jovian ionosphere. This quantity corresponds to the width of the Io flux tube footprint.

lies between 10 and $1000 \mathrm{~s}$. As far as the UV and visible footprint counterparts are concerned, two families of results have been published. On the one hand, the studies based on the Faint Object Camera (FOC) on board the Hubble Space Telescope (HST) at far UV wavelength report the footprint spot to be $400(-200,+100) \mathrm{km}$ long [Prangé et al., 1996, 1998]. Similarly, Vasavada et al. [1999], described the Io footprint as a $450 \pm 100 \mathrm{~km}$ diameter circular patch, based on visible images acquired with the SSI instrument on board the Galileo spacecraft. These results lead to the conclusion that the IFP size roughly corresponds to the projected size of Io in the Jovian ionosphere (see Figure 1 for more details). On the other hand, Clarke et al. [1996], using the second generation Wide Field and Planetary Camera 2 (WFPC2), measured the Io footprint spot to be as long as 1000 to $2000 \mathrm{~km}$, much larger than Io's projected diameter. Later studies making use of the third-generation Space Telescope Imaging Spectrograph (STIS) instrument showed that the Io spot can even be as long as $15^{\circ}$ longitude [Clarke et al., 2002]. The authors explained the discrepancy with the FOC-based measurements by the lack of sensitivity of this instrument, which would only be able to detect the brightest part of the emission. Clarke et al. [2002] also found that the FUV emissions related to Io could sometimes appear as a pair of spots instead of a single one. These spots were found to be a few thousand kilometers apart at maximum. These authors noted that this length could corresponds to the time required for a field line interacting with Io to be brought back to corrotation in the 7 Io radii long stagnant plasma wake [Hinson et al., 1998]. Consequently, they interpreted the brightest part of the Io footprint, no matter if is formed by one large spot or by multiple spots, as the signature of an interaction region consisting of Io and its plasma wake. On the contrary, Gérard et al. [2006] considered the different spots as distinct features and studied the spots' multiplicity on a larger set of STIS images. By isolating the main spot, they measured its typical length to be $\sim 0.9^{\circ}$ of longitude. Serio and Clarke [2008] also measured the Io footprint size, but in a quite different way. They estimated the footprint diameter from the full width at half maximum (FWHM) of the footprint perpendicular to the footpath direction. They found that this quantity ranges from 500 to $2000 \mathrm{~km}$ and that it is not correlated with the centrifugal latitude of Io, i.e., with the latitude of Io with respect to the torus's centrifugal equator. Finally, Gladstone et al. [2007] measured the main spot width to be $400 \mathrm{~km}$, based on images acquired with the LORRI visible imager on board New Horizons. They also found that the vertical extent of the main spot appears as long as $1000 \mathrm{~km}$.

[4] From the above review of the different numbers for the Io footprint size, it appears that the "Io footprint size" appellation is sometimes used to designate different concepts. The Io footprint main spot is an emission volume which has a length (horizontal and along the footpath), a vertical extent and a width (horizontal and perpendicular to the footpath direction), as shown on Figure 2. Depending on the orientation of the spots on the image, the measurable "size" of the footprint is usually a combined projection of these three quantities, with the exception of some particular cases.

[5] These cases are here extensively used by identifying subsets of images showing the spots from specific points of view. The present work is thus divided into three parts, one for each dimension of the spots. When the spots lie close to the central meridian line, the footprint is generally seen from the side: a favorable configuration to estimate the lengths along the footpath. The length of both the MAW and the TEB spots are discussed in section 3.1. Moreover, Bonfond et al. [2009] have recently shown that it is possible to measure the vertical extent of the Io footprint tail emissions by detecting the point where the tail is right above the limb plane. Unfortunately, no image has been acquired in a configuration such

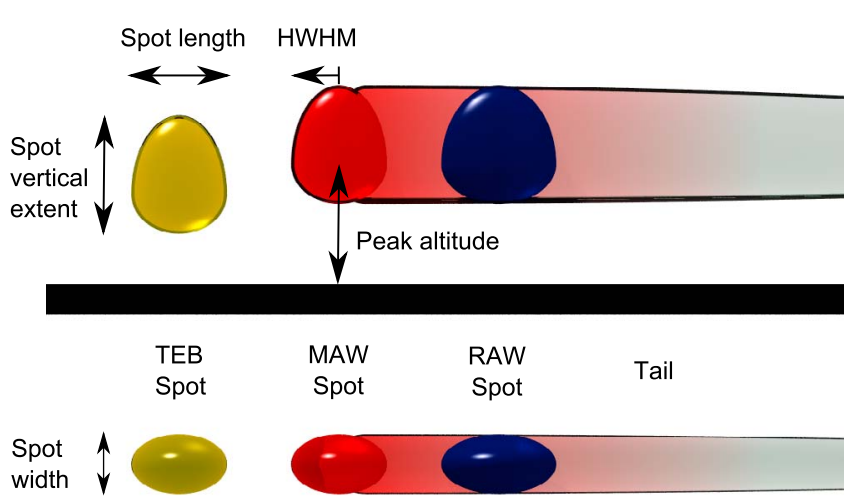

Figure 2. Sketch of the Io footprint as seen (top) from the side and (bottom) from above. The Io footprint is considered to be formed by three spots and an extended downstream tail. The actual length of the MAW spot cannot be directly measured in practical cases because its downstream part overlaps with the tail and sometimes with other spots. It has thus been chosen to characterize the spot length through its upstream half width at half maximum (HWHM). Note that each spot has its own set of parameters (length, peak altitude, etc.), but in the present study, they are all assumed to have the same width. 


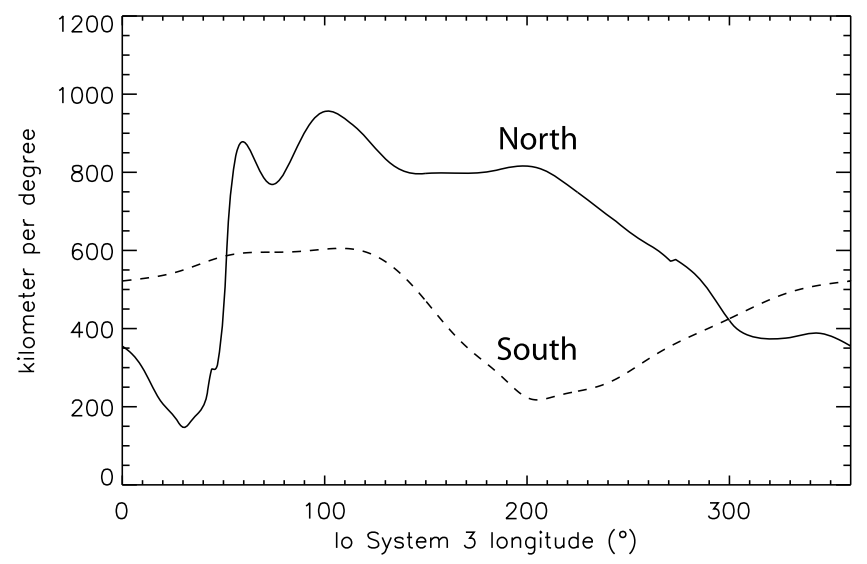

Figure 3. Variation of the number of kilometers per longitude degree measured on the planet as a function of the System III longitude of Io, based on the reference footpath of Bonfond et al. [2009]. The solid line corresponds to the north, while the dashed line corresponds to the south.

that the spots are exactly in the limb plane. However, there is a way to circumvent this issue which is discussed in section 3.2. Finally, some particular IFP curtain configurations, observed when the spots are aligned with the line of sight, are best suited to measure the footprint width. This measurement is the most difficult one and is described on section 3.3.

\section{Data Processing}

[6] The present study is based on FUV images of Jupiter's aurora acquired with the Hubble Space Telescope's Space Telescope Imaging Spectrograph (STIS) and Advanced Camera for Surveys (ACS) instruments between 1997 and 2009. The far-ultraviolet (FUV) channels of both instruments use the same type of multianode microchannel array detectors. FUV auroral emissions on Jupiter originate from $\mathrm{H}_{2}$ Lyman and Werner bands as well as from the $H$ Lyman- $\alpha$ line. However, Lyman- $\alpha$ emissions are usually contaminated by geocoronal emissions. This study is based on images acquired with filters including the Lyman- $\alpha$ line (i.e., the clear filter on STIS and the F115LP filter on ACS) or rejecting it for an improved signal to noise ratio (i.e., the strontium fluoride filter on STIS and the F125LP filter on ACS). Two observation modes have been used for STIS observations: the standard ACCUM mode and the time-tag mode, for which not only the location of the counts are registered, but also their arrival time. Each time-tag sequence has been organized into $10 \mathrm{~s}$ intervals. All the counts detected during a $10 \mathrm{~s}$ interval are gathered to create an image. Consequently, the time-tag event list is reduced to a sequence of $10 \mathrm{~s}$ exposure time consecutive images. The duration of these time-tag sequences can vary from $120 \mathrm{~s}$ up to $2700 \mathrm{~s}$, i.e., one complete HST orbit, for the GO-11649 observation campaign that took place in summer 2009. Some of the methods used to determine the size of the footprint spots require an automatic determination of the location of the MAW spot on the images, and thus they require the knowledge of the position of Jupiter's center on each image. The planetary centers have been determined with the same automatic limb fitting routine as described by
Bonfond et al. [2009], which has an expected uncertainty of $\sim 1$ pixel.

\section{Observations}

\subsection{Io Footprint Spots Length}

\subsubsection{Method and Units}

[7] In the introduction, footprint lengths have been expressed both in degrees of longitude and in kilometers. However, one degree of longitude measured at one point along the footpath can correspond to a very different distance in kilometers than at another point, because of the offcentered and distorted Io footpath shape, especially in the north. One definitive way to deal with the problem would be to map the distances along the magnetic field lines. Unfortunately, the accurate measurement of the footprint positions sheds some doubts on the ability of the present magnetic field models [Connerney et al., 1998; Grodent et al., 2008] to perform reliable mappings [Bonfond et al., 2009].

[8] Consequently, only the comparison between distances measured in degrees on the planet and distances measured in kilometers is considered here. The distance which corresponds to one degree of longitude on the planet depends on the System III longitude and is represented on Figure 3. The mean values are $625 \mathrm{~km} / \mathrm{deg}$ in the north and $497 \mathrm{~km} / \mathrm{deg}$ in the south. It should be noted that the conversion coefficient can varies with the Io longitude by a factor of $\sim 5$ in the north and $\sim 2$ in the south. As a result, there is no unique coefficient to convert degrees to kilometers.

[9] The first step for the length measurement consists in selecting images where the MAW spots are less than 30 degrees away from the central meridian line, so that the Io footprint can be seen from the side. Groups of 10 timetag sub-images are summed to form standard $100 \mathrm{~s}$ images. The data set for this part of the study includes 263 images of the northern hemisphere and 199 images of the southern hemisphere. For each image, an Io footprint stripe is automatically extracted according to the reference footpath from Bonfond et al. [2009]. The next step consists in removing the local planetary disk background close to the IFP spots. Then, each stripe starts $4000 \mathrm{~km}$ upstream of the tabulated MAW spot position. Every $25 \mathrm{~km}$ in the downstream direction, the corresponding pixel is located on the image and its brightness is reported. This procedure is repeated for altitudes ranging from the 1 bar level $(0 \mathrm{~km})$ to an altitude of $2000 \mathrm{~km}$. In other words, each stripe consists of a stack of brightness distributions along the reference Io footpath measured on ellipsoids of differing radii. Once the stripes are formed, the brightness is summed vertically to generate brightness profiles. The profiles are considerably more extended in the downstream direction because the tail emissions, and sometimes downstream spots, add to the main spot. Measurements of the profiles' full length at half maximum cannot be used directly since they are usually contaminated with emissions unrelated to the MAW spot. Instead, the upstream half length at half maximum (HLHM) is chosen to characterize the length of the main spot as if it were isolated from the other IFP emissions. The actual length is then assumed to be twice the measured HLHM, as presented in Figure 4. Note that northern hemisphere measurements are only available between $110^{\circ}$ and $230^{\circ}$ System III longitudes. The results, when expressed in kilometers, are 


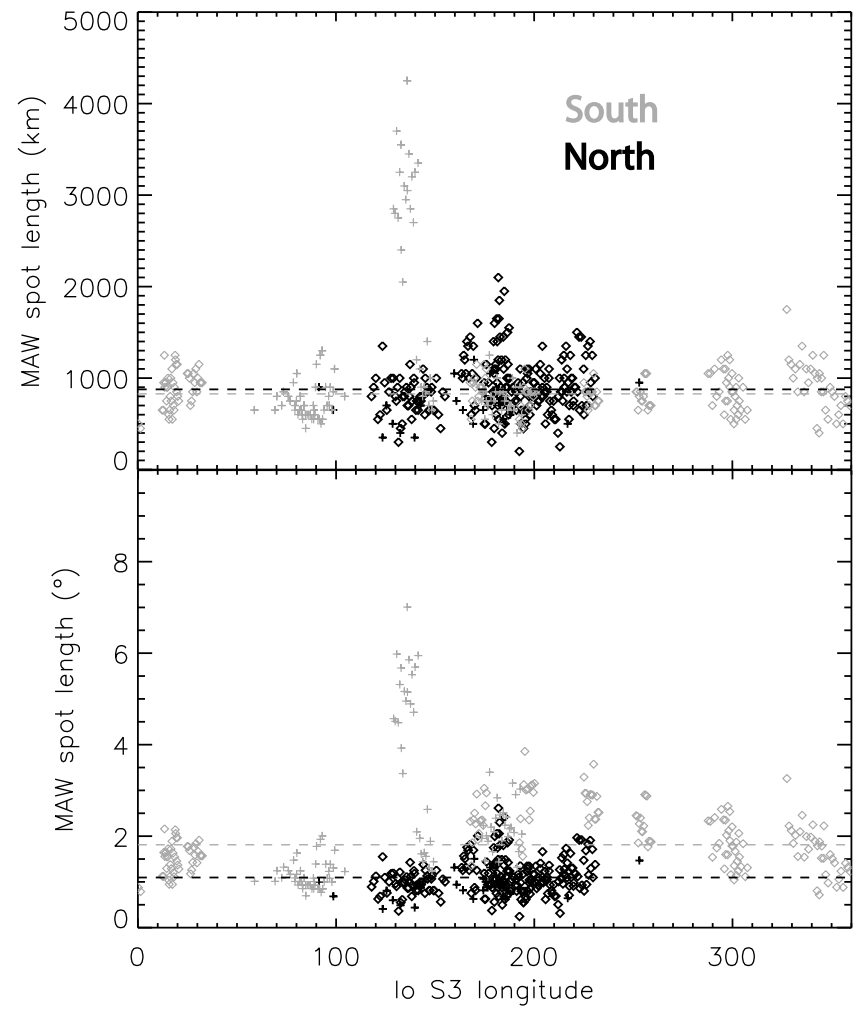

Figure 4. Measured length of the Io footprint main Alfvén wing spot as a function of the Io System III longitude. The top plot is for length measured in degrees, while the bottom plot is for measurements in kilometers. The data points corresponding to STIS measurements are represented with the plus symbol, while ACS data are noted with diamonds. Black symbols are for measurements in the northern hemisphere, while gray symbols are measurements in the southern hemisphere. The length of the MAW spot is defined as twice the upstream half width at half maximum. The dashed lines represent the mean values for the length in the northern hemisphere (black) and the southern hemisphere (gray). In the south, the points between $125^{\circ}$ and $145^{\circ}$ Io System III longitude have been excluded to compute the means, since the measured length does not correspond to the MAW spot length in this range. As detailed in section 3.1, the expected full-length uncertainty for individual points is of the order of $400 \mathrm{~km}$.

relatively constant for all Io longitudes and for both hemispheres if we exclude the southern data points between $125^{\circ}$ and $145^{\circ}$ Io System III longitude (see section 3.1.2).

[10] The mean length is $\sim 850 \mathrm{~km}(\sim 880 \mathrm{~km}$ for the north and $\sim 830 \mathrm{~km}$ for the south) and is independent of the filter. Nevertheless, it should be noted that the drawback of the method is a relatively large uncertainty, since the standard deviation is around $260 \mathrm{~km}(\sim 300 \mathrm{~km}$ for the north and $\sim 210 \mathrm{~km}$ for the south). Assuming a 1 pixel uncertainty $(\sim 100 \mathrm{~km})$ for the brightness peak location and an equivalent uncertainty for the half-maximum point location, the total uncertainty is of the order of $\sim 200 \mathrm{~km}$ for the HLHM and thus of $\sim 400 \mathrm{~km}$ for the full length for each individual case. On the basis of Figure 3, it should be noted that the reported length is in accordance with the $0.9^{\circ}$ measured by
Gérard et al. [2006]. However, when expressed in degrees of longitude, the mean MAW spot length is twice larger in the south than in the north. Additionally, the southern lengths expressed in degrees significantly vary with the Io longitude while values expressed in kilometers do not. Consequently, kilometers are preferentially used when comparing Io footprint characteristics.

\subsubsection{Contiguous Spots}

[11] In Figure 4, the STIS southern hemisphere data points between $125^{\circ}$ and $145^{\circ}$ have considerably larger values than the other data points. These points are not outliers but correspond to particularly long footprints like the one shown in Figure 5 (stripe c). The plots clearly demonstrate that these cases are atypical and should be considered with caution when drawing conclusions on the spots length, and the reason for these atypically high values is now discussed. During summer 2009, three HST observation orbits were dedicated to the observation of the southern footprint when Io was moving through the central region of the torus from south to north. The evolution of the morphology as a function of the System III longitude of Io is shown in Figure 5. The downstream TEB spot approaches the MAW spot until they practically merge at the end the first sequence. When the TEB spot emerges upstream from the MAW spot while the RAW spot is only around $1500 \mathrm{~km}$ downstream, the combination of the three spots appears as one very large spot. Note that this particular shape strongly depends on the relative brightness of the three spots and the tail. Figure 5 shows the results of a simulation of the evolution of three Gaussian-shaped spots and an exponentially decreasing tail when the TEB spot moves from downstream to upstream. For a similar configuration, the resulting modeled footprint has a length up to three times larger than the typical MAW length, which is similar to the maximum value shown in Figure 4.

[12] As a conclusion, the elongated footprints observed in the southern hemisphere between $120^{\circ}$ and $150^{\circ}$ are not necessarily the image of an extended interaction region at Io which comprises the stagnating plasma wake. Instead, based on simulations, I argue that these peculiar morphologies are the consequence of the contiguity of the different spots in this particular sector.

\subsubsection{Motion Blur and Io Flux Tube Length}

[13] The observationally derived typical length should be compared with two quantities: the distance covered by the MAW spot on the image during a typical exposure time and the projected Io diameter along magnetic field lines. Figure 6 displays the length covered by the IFP in a reference frame fixed in local time for a typical $100 \mathrm{~s}$ exposure time. The values plotted here should be projected relative to the image viewing angle to provide the actual IFP motion on the image. However, this latter step is not necessary for the present discussion, because the projection effect on distances along the footpath is minimum when the footprint is observed close to the CML. The displacement of the IFP during the exposure time generates a motion blur which could affect the measured footprint length. In the southern hemisphere, the covered distance ranges from $\sim 200 \mathrm{~km}$ to $\sim 350 \mathrm{~km}$, while in the North, the distance varies from $\sim 50 \mathrm{~km}$ to $\sim 800 \mathrm{~km}$. This latter value is very close to the measured length. Nevertheless, modelling the MAW spot brightness profile with a Gaussian function and convolving it with a step function as long as the original Gaussian 

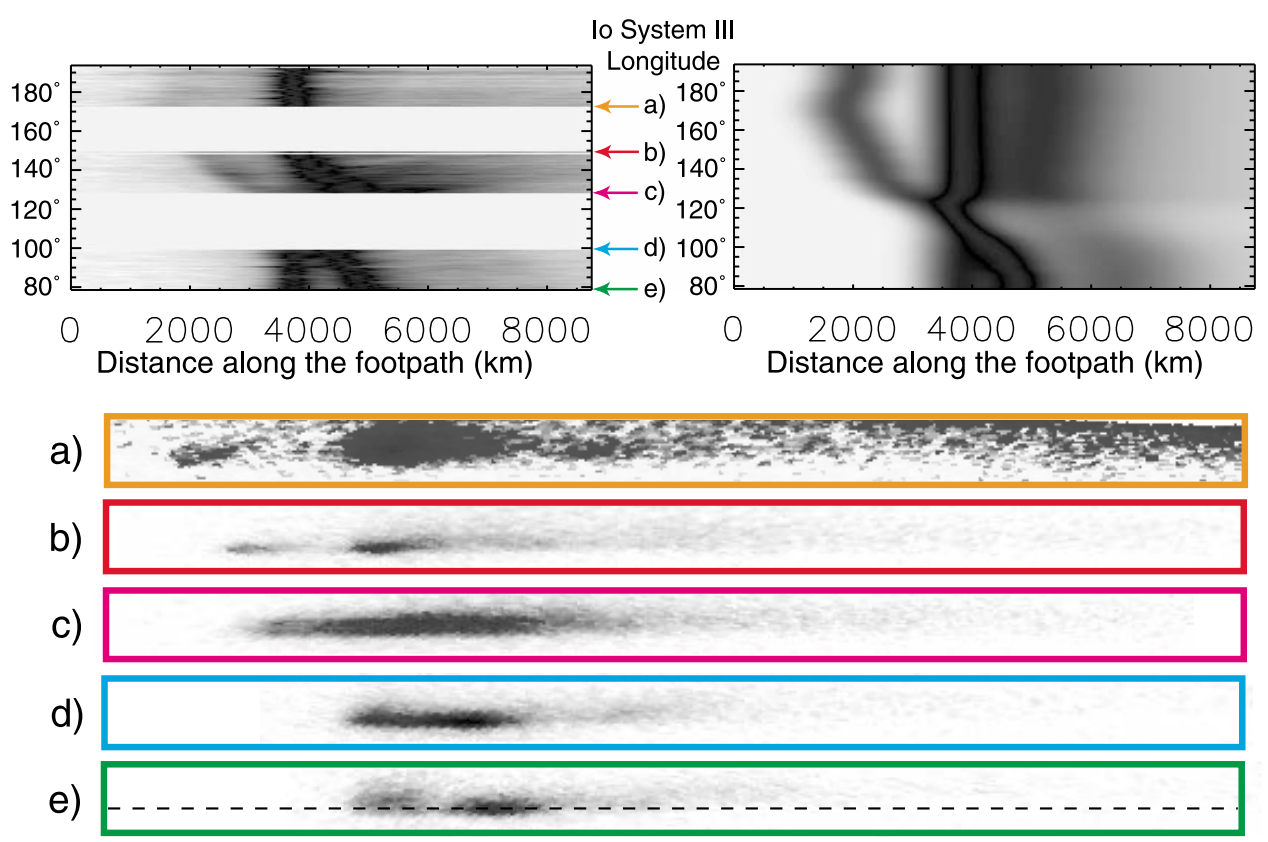

Figure 5. (top left) Evolution of the southern footprint morphology when Io moves from $78^{\circ}$ to $194^{\circ}$ System III longitude. Each line represents the evolution of the Io footprint relative brightness for one image extracted from the three time-tag sequences acquired in the southern hemisphere in 2009. (top right) Simulation of the evolution of the Io footprint morphology, assuming three Gaussian spots and an exponentially decreasing tail. Setting the relative spot brightness apart, the morphology is very well reproduced. The configurations where the footprint appears as a very large spot correspond to cases where the MAW and the TEB spots are close to each other. (bottom) Selected Io footprint stripes corresponding to the profiles shown on the top left plot. The contrast has been enhanced for stripe a in order to highlight the faint TEB spot. The dark shape on the top of this stripe is an artifact from the main auroral oval. The dashed line in stripe e is drawn to highlight the shift between the MAW spot (upstream) and the TEB spot (downstream).

full length at half maximum (FLHM) increases the apparent FLHM by $20 \%$ only. However, this calculation assumes that the spot's brightness remains constant during the exposure time (typically $\sim 100 \mathrm{~s}$ ). Bonfond et al. [2007] showed that the footprint spots can experience brightness variations of the order of $50 \%$ on timescales of one minute and this could also affect the apparent length. In the worse case scenario, the spot brightness first decreases by a factor of two until the middle of the exposure and then increases again up to the maximum. In this case, the length increases by approximately $30 \%$. Thus, even in the most critical cases, the length measured on 100 s exposure images is only moderately affected by the motion blur. Furthermore, in the present study no measurements have been made in these critical areas of the northern hemisphere where the MAW spot length is difficult to observe. If the covered distance is one half of the initial Gaussian FLHM (i.e., $\sim 450 \mathrm{~km}$ ), the apparent length increases by $7 \%$ at maximum, which is below the measurement uncertainties. Consequently, the apparent size of the MAW spot cannot be attributed to the motion blur.

[14] Figure 1 shows the size of the unperturbed Io flux tube footprint in the northern and the southern hemispheres according to the VIP4 magnetic field model. The IFT length varies from $\sim 200 \mathrm{~km}$ to $\sim 350 \mathrm{~km}$. The FWHM of the STIS and ACS point spread functions are barely larger than two and one pixels, respectively. Accordingly, the widening of the spot is not an instrumental effect. As a consequence, the MAW spot length shown on Figure 4 appears to be 3-4 times more elongated than the expected flux tube length. This result does not necessarily imply that the interaction region at Io is three to four times as large as Io. The plasma interaction between Io and the Jovian magnetosphere implies a piling up of the field lines at Io. Therefore it is expected that the actual (i.e., perturbed) flux tube length is larger than the unperturbed one because the portion of the flux tube that interacts with Io is considerably slowed down while the remaining continues to rotate at its initial speed [Kivelson et al., 2004; Saur et al., 2004]. Additionally, the spot is not located at the foot of the Io flux tube but rather at the foot of the current carrying Alfvén wing. Jacobsen et al. [2007] showed that nonlinear effects on the Alfvén waves' propagation can considerably increase the length of the Alfvén wing. Moreover, Jacobsen et al. [2010] showed that such nonlinear effects are necessary to explain the presence of electron beams close to Io as observed by Galileo.

\subsubsection{Length of the TEB Spot}

[15] The length of the TEB spot can also be easily measured with the same technique as the MAW spot if it is (1) located upstream of the MAW spot, (2) oriented perpendicularly to the line of sight, and (3) bright enough. These three conditions are only fulfilled in the southern hemisphere when Io's System II longitude lies between $130^{\circ}$ and $260^{\circ}$. 


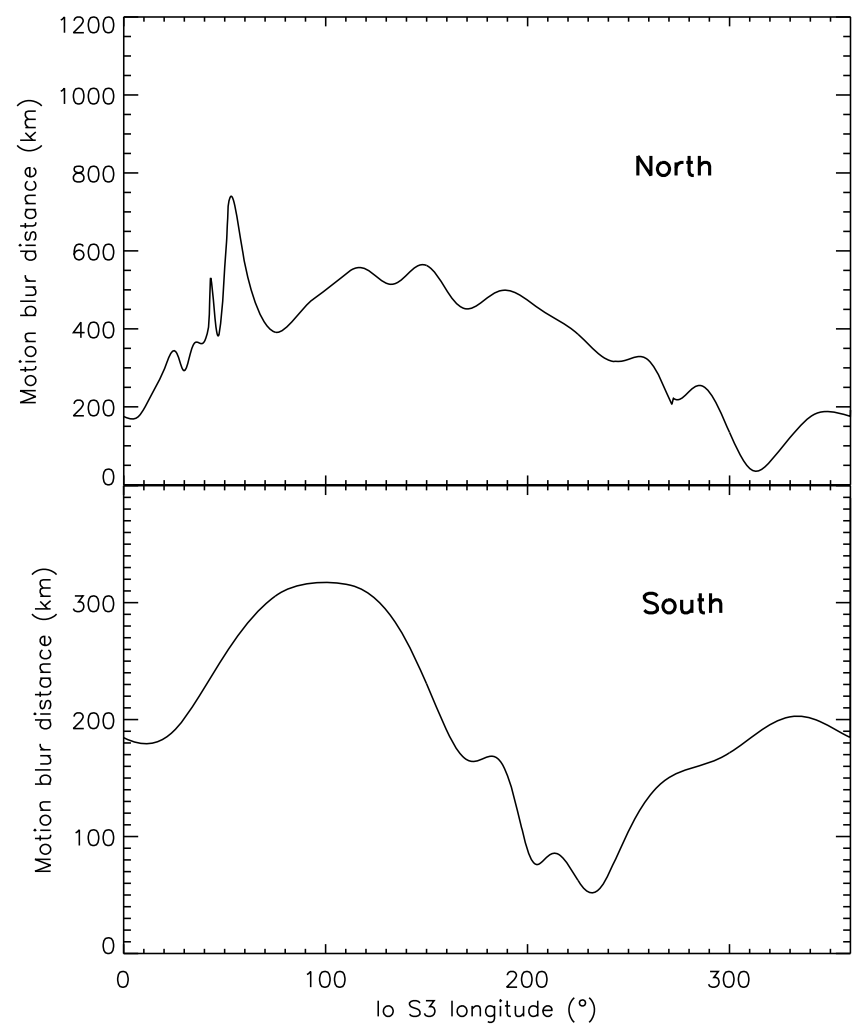

Figure 6. Motion blur distance for an exposure time of $100 \mathrm{~s}$ as a function of the Io System III longitude. The motion blur is the distance covered by the MAW spot in a reference frame fixed in local time.

On the basis of analysis of 92 images, the TEB spot length is $\sim 860 \pm 150 \mathrm{~km}$ and does not significantly vary with Io's System III longitude.

\subsection{Io Footprint Spots Vertical Extent}

[16] Bonfond et al. [2009] used images where the tail emissions are observed above the Jovian limb, which is assumed to correspond to the 1 bar level. Here, only a subset of these images is considered, for which the spots are also observed above the limb. The observations have been acquired in Spring 2007 during 14 HST observation orbits with the ACS instrument. Contrary to the tail case, no image has been acquired with one spot exactly in the limb plane. Consequently, the apparent altitude of the main spot emission is not its actual altitude and thus another strategy is required to retrieve it. Since the longitude and the latitude of the main spot are known from the IFP reference footpath, the expected MAW position on the image can be computed if its real peak altitude is the same as for the tail (i.e., $\sim 900 \mathrm{~km}$ ). If the observed peak altitude significantly differs from the simulation, this would indicate that the main spot is not at the assumed altitude. Consequently, the method consists in generating an image of the tabulated Io footpath for a fixed altitude of $900 \mathrm{~km}$, assuming the actual viewing geometry and in comparing it to the observation.

[17] Similarly to Bonfond et al. [2009], image data are gathered as sets of three successive images. Then radial scans are performed on the Io footprint area of the summed image. For the present study, the objective is to identify the position of the MAW spot by locating maximum brightness. Figure 7 shows an example of the comparison between the observed and the simulated peak altitude as a function of the scan angle. The mean difference between the modeled and the observed altitudes is $85 \mathrm{~km}$ with a standard deviation of $155 \mathrm{~km}$. Since these quantities are of the order of the pixel size $(\sim 100 \mathrm{~km})$, it is reasonable to conclude that the MAW spot peak altitude is similar to the tail peak altitude, which confirms results from color ratio measurements [Gérard et al., 2002].

[18] An important result from Bonfond et al. [2009] was that the vertical scale height of the tail emission is so large that its width can be modeled only by assuming a kappa energy distribution for the incoming electrons. This result favors acceleration mechanisms associated with inertial Alfvén waves [Hess et al., 2010]. Consequently, the shape of the emission vertical profile of the MAW spot is also expected to provide us with information on the acceleration mechanism. Chapman profiles of the form

$$
f(x)=C \exp \left(1-\left(\frac{Z-Z_{0}}{H}\right)-\exp \left(-\frac{Z-Z_{0}}{H}\right)\right),
$$

where $C$ is a constant, $Z$ is the altitude in $\mathrm{km}, Z_{0}$ is the altitude of the peak in $\mathrm{km}$ and $H$ is the scale height in $\mathrm{km}$, have been fitted to the observed curve. The resulting mean scale height is $366 \mathrm{~km} \pm 53 \mathrm{~km}$ and does not depend on the filter. These values are similar to those observed for the tail $(430 \mathrm{~km} \pm 70 \mathrm{~km})$. Additionally, a Chapman profile with such a scale height has a full width at half maximum of $\sim 850 \mathrm{~km}$, which is consistent with estimates by Gladstone et al. [2007]. As a conclusion, the precipitating electrons generating the IFP main spot appear to have the same characteristics as for the tail, i.e., a kappa energy distribution with a mean energy $\sim 1-2 \mathrm{keV}$. The neutral atmosphere profile used to derive this value comes from the auroral atmospheric model of Grodent et al. [2001]. A detailed study of the sensibility of the computed energy distribution as a function of the atmospheric profile is given by Bonfond [2009]. This analysis showed that the typical energies could only vary by a factor of $\sim 4$ at maximum.

[19] On one single orbit, TEB spot emissions can also be observed above the limb (Figure 7). As discussed above, the observed and the modeled altitudes are fairly similar for the tail and for MAW spot, but the observed altitude for the TEB spot appears to be $\sim 200 \mathrm{~km}$ lower. A Monte Carlo model of the electron energy degradation in the Jovian atmosphere can then be used to derive the typical energy required to produce emissions at this altitude [Bonfond et al., 2009]. The corresponding mean electron energy would then be approximately twice more energetic than for the MAW spot and for the tail, whatever the considered energy distribution. Unfortunately, the low number of favorable cases and the weakness of the TEB spot do not allow us to use reliably the complete vertical profile to retrieve the energy distribution. The fact that the TEB emissions peak at a lower altitude could also explain some misalignment between the TEB spot and the remainder of the tail. An example of such a misalignment of the TEB spot relative to the MAW spot and 

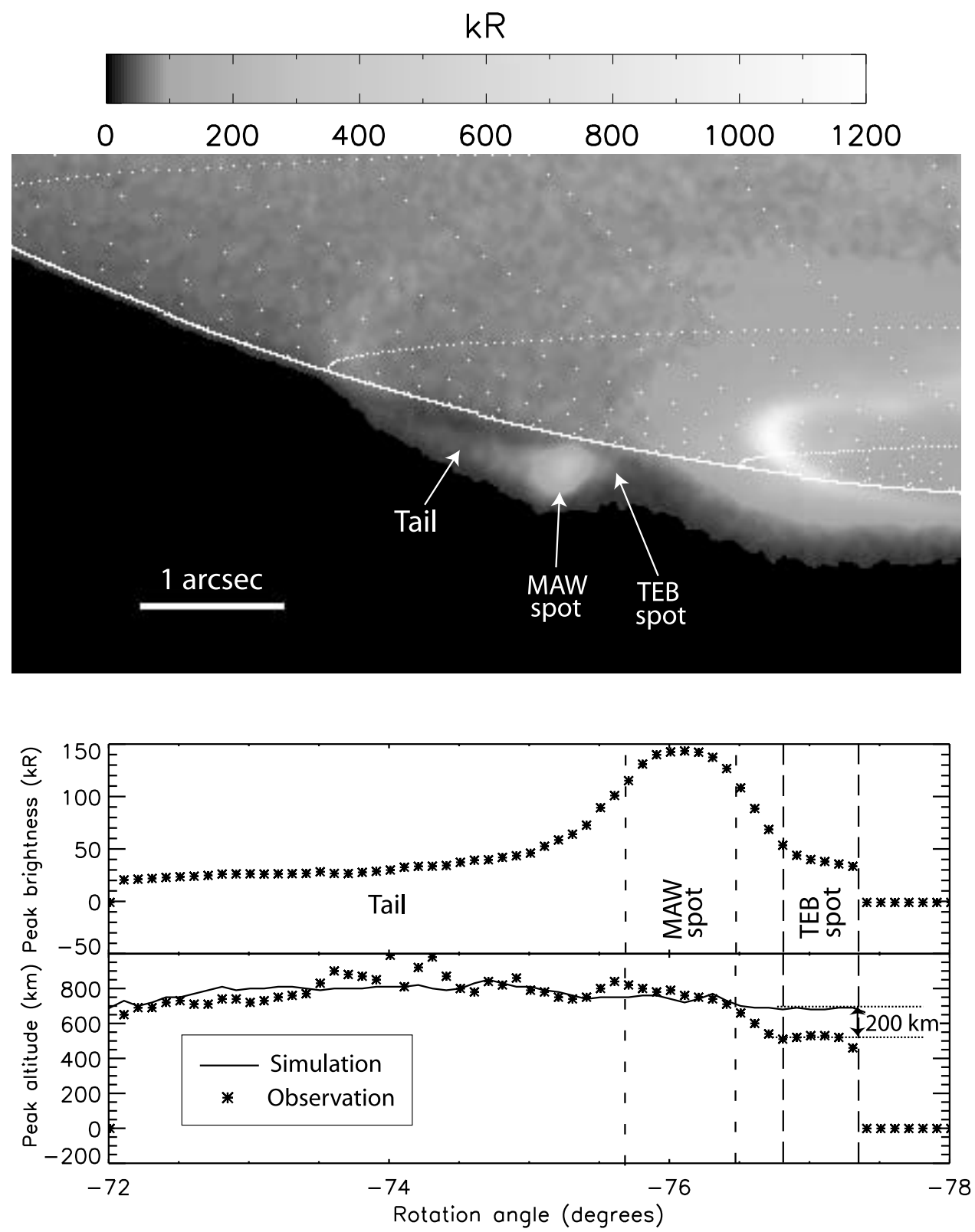

Figure 7. (top) Example of tail and spots observed above the limb. (bottom) Profiles of the peak brightness and the peak altitude as a function of the rotation scan angle (stars). $0^{\circ}$ corresponds to the equator, and $-90^{\circ}$ corresponds to the South Pole. The expected apparent altitude of an Io footpath located at $900 \mathrm{~km}$ is represented in solid line. The MAW spot corresponds to the peak in the brightness profile. The observed and the predicted apparent peak altitudes match fairly well along the tail as well as in the MAW spot region. In this example, the bottom plot shows that a faint TEB emission also appears upstream of the MAW spot. The motion of this emission on consecutive images from the same HST orbit unambiguously associates it with the Io footprint and not with the main aurora. Its apparent altitude is $200 \mathrm{~km}$ lower than expected for a spot located at $900 \mathrm{~km}$.

the tail can be seen on Figure 5e. This slight shift could be interpreted either as a latitudinal shift or as an altitude shift. The new observations presented here clearly favor the latter.

\subsection{Io Footprint Width}

[20] The actual Io footprint width can be estimated only if two conditions are fulfilled. First, the viewing angle should be such that the main spot is aligned with the line of sight. Second, the exposure time should be short enough to avoid significant motion blurring of the IFP position. While insignificant for the spot length, the displacement of the footprint during the exposure cannot be neglected for the width. This latter constraint imposes the use of images derived from timetag sequences.

[21] Similarly to the method applied to estimate the spots length and the vertical extent, the first step consists in identifying images where the footprint is in such a configuration that the main spots and the beginning of the tail are suitably 

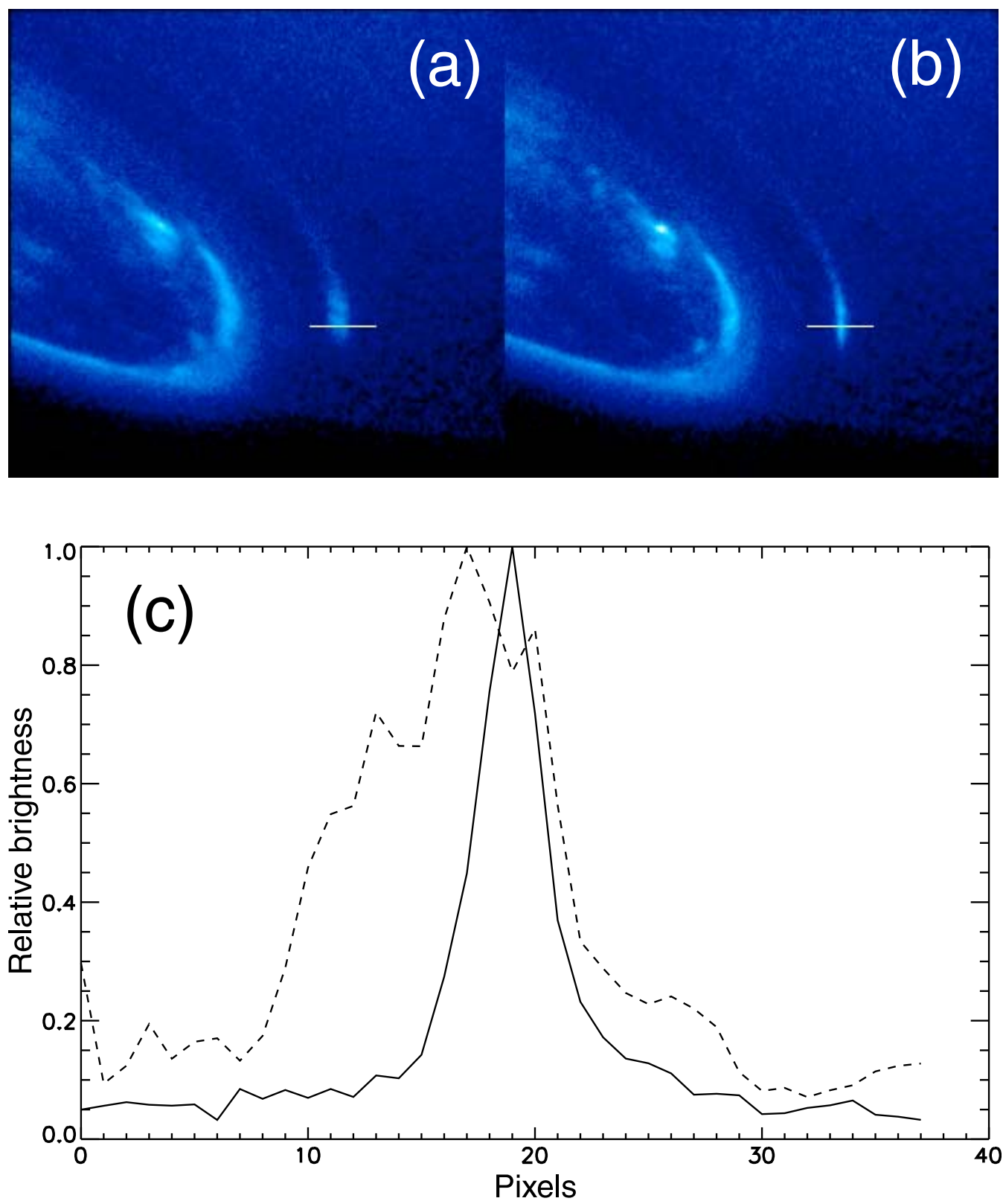

Figure 8. Example of Io footprint as seen from above (a) without and (b) with motion compensation. (c) Comparison of the apparent width of the auroral curtain with (solid line) and without (dashed line) motion compensation.

oriented. Unfortunately, the number of suitable time-tag sequences is limited to two. In order to avoid the motion blur which affects the width while keeping a sufficient signal to noise ratio, the $10 \mathrm{~s}$ frames were manually shifted in order to compensate for the IFP motion before being co-added.

[22] Figure 8 shows an example of images generated from the same sequence with and without motion compensation. It is noticeable that the motion obviously increases the IFP width, which directly indicates that the instantaneous IFP curtain width is significantly smaller than the motion blur extent. After removing the local planetary background close to the spots, the width is measured by extracting a brightness profile perpendicular to the footpath. However, the width of this profile is strongly dependent on the orientation of the footprint on the image, and thus does not represent the curtain width. Actually, even in the very restrictive case selection used here, the footprint is never exactly aligned with the observer. Therefore it is necessary to model the real image geometry to take these orientation effects into account. Consequently, a 3-D IFP emission model has been built in order to demonstrate the existence of these effects and evaluate their impact. This model simulates the apparent IFP width for different actual input width. It consists in simulating the FUV optically thin emissions for an IFP formed of three independent spots and an extended tail. The longitude and latitude of the spots and the tail are derived from the reference footpath and interspot distances reported by Bonfond et al. [2009]. For each feature, a law describing the evolution of the brightness 

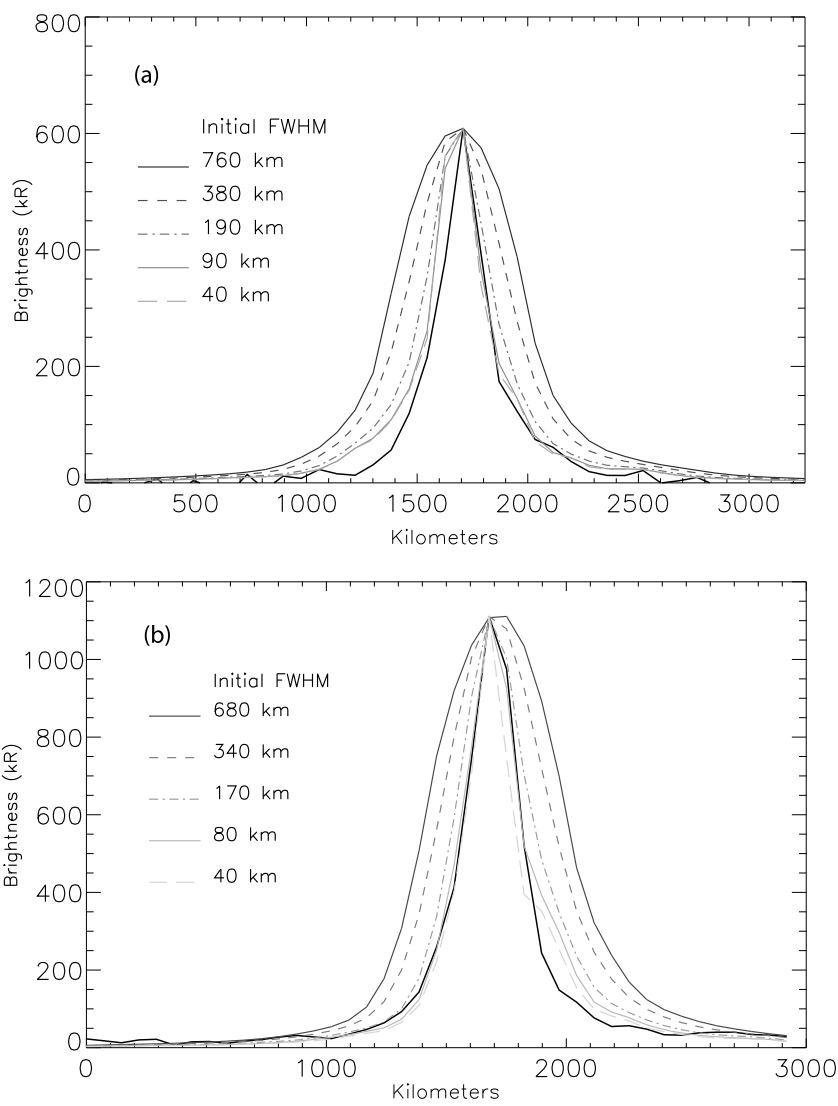

Figure 9. Comparison of measured Io footprint width profile (bold) with simulated profiles calculated for different footprint widths. Both cases are from the northern hemisphere. The Io System III longitude is $209^{\circ}$ in the first case (Figure 9a) and $145^{\circ}$ in the second one (Figure 9b).

along the footpath, across the footpath, and vertical to the planetary surface are required. The vertical profiles are modeled with Chapman profiles set according to the peak altitude and scale height measurements discussed above. The variations of the brightness along the length and the width directions follow a Gaussian law. The spots' full length at half maximum is set to $850 \mathrm{~km}$, but the full width at half maximum is a free parameter. Among the unavoidable hypotheses on the IFP geometry, this simulation assumes that all the IFP features, i.e., the three spots under consideration and the tail, have the same width. As far as the tail is concerned, the brightness initially increases along the footpath as a Gaussian law and then evolves as a decreasing exponential law with a $21,000 \mathrm{~km}$ e-folding distance [Bonfond et al., 2009]. Knowing the 3-D location and the brightness of each point of the IFP, emissions can then be integrated along the line of sight to generate a simulated image. The simulation grid is initially over-sampled twice compared to the original pixel size. Then, the pixels are rebinned and the resulting modeled image is convolved with the instrumental point spread function (PSF). Figure 9 shows a comparison between modeled and measured widths.

[23] In the two suitable observations, the observed profile is as thin or even slightly thinner than the thinnest model profile. Reducing the input width below a certain threshold has a minor impact on the model output because of the finite pixel size and PSF size as well as the misalignment of the footprint. As long as the simulated FWHM input remains less than $\sim 170-190 \mathrm{~km}$, the modeled widths remain fairly similar to the measured ones. Significant differences only arise when this threshold is exceeded. As a result, only an upper limit of $\sim 200 \mathrm{~km}$ can be estimated for the IFP width.

[24] The expected projection of Io's width along VIP4 magnetic field lines lies between 100 and $160 \mathrm{~km}$ (Figure 1). Contrary to the spots length, no interaction model predicts a significant increase of the IFP width compared to the projected Io width. Consequently, the diameter of the interaction region measured perpendicularly to the plasma stream does not exceed twice the size of Io.

\section{Discussion and Conclusions}

[25] The size of the Io footprint is an important issue in understanding the Io-Jupiter interaction. On the basis of the apparent limited size of the Io footprint on some images, some studies suggested that the interaction is limited to Io's immediate vicinity. On the other hand, its extended length as seen on other images led other authors to reach the opposite conclusion: i.e., the interaction region should be as large as the stagnating wake observed downstream of Io. The present work takes advantage of the increased number of images at both high resolution and high sensitivity to examine these contradictory results.

[26] In order to provide physically significant values to characterize the Io footprint size, its 3-D structure has to be properly understood. First, the different spots are distinct features that must be considered separately, as shown by Gérard et al. [2006] and Bonfond et al. [2008]. Second, each feature has a length along the footpath, a vertical extent and a width. Therefore each image is a 2-D projection of a three dimensional emission region. It is thus necessary to carefully select subsets of images where the spots are seen from the side, above the limb or aligned with the line of sight, respectively.

[27] The lengths of both the main Alfvén wing (MAW) spot and the trans-hemispheric electron beam (TEB) spot are of the order of $850 \mathrm{~km}$. These values are not an artifact of the motion of the spot during the exposure nor caused by the instrumental PSF. Focusing on the main spot, we see that the length is significantly larger than the size of Io projected along unperturbed magnetic field lines but could correspond to the projected size of the stagnating plasma wake. However, the magnetic field lines are indeed perturbed by the presence of Io and the subsequent broadening of the main spot length along the footpath is expected from simulations, even if the core of the interaction remains relatively close to the satellite [Jacobsen et al., 2007]. Additionally, the large spot sizes observed by Clarke et al. [2002] are untypical and can be attributed to the combination of the different spots in these configuration. The $0.9^{\circ}$ long MAW spots as measured by Gérard et al. [2006] correspond much more likely to the actual typical length. In any case, the conversion from degrees to kilometers is not unique but strongly depends on the Io System III longitude. The small length of the footprint, as estimated from visible instruments [Vasavada et al., 1999; Gladstone et al., 2007] and from the FOC camera [Prangé et al., 1996, 1998], is most probably a consequence of their limited sensitivity. 
[28] As far as the MAW spot vertical extent is concerned, no obvious difference is observed compared to the tail emissions. The peak altitude lies around $900 \mathrm{~km}$ and the scale height is approximately $350-400 \mathrm{~km}$. As a result, the mean precipitating electron energy is of the order of $1-$ $2 \mathrm{keV}$ for the auroral atmospheric profile of Grodent et al. [2001]. Additionally, the broadness of the impinging electrons energy distribution suggests that inertial Alfvén waves cause the electron acceleration [Bonfond et al., 2009]. This conclusion is nevertheless less surprising for the MAW spot than it was for the tail, since a steady state is expected to be established only downstream of the spots. The TEB spot peak altitude appears to lie at $700 \mathrm{~km}$, implying that the mean electron energy is twice higher than for the MAW spot. The different peak altitude for the MAW and the TEB spot concurs with the scenario of the trans-hemispheric electron precipitation. Such a difference in the electron energy distribution has been predicted by the inertial Alfvén wave electron acceleration model described by Hess et al. [2010]. These authors showed that the perpendicular Alfvén wave length scale could control the direct and the conjugate precipitated energy distribution. Moreover, it is noted that the quantity measured by Serio and Clarke [2008] is probably not the actual width of the IFP but the projection of its vertical extent, which is strongly dependent on the viewing angle.

[29] The footprint width is by far the most difficult spot quantity to measure. The method used in this study only provides an upper limit $(\sim 200 \mathrm{~km})$ but this number is also consistent with an interaction region restricted to the immediate Io neighborhood. The narrowness of the footprint curtain is an important result of this work. The Far UV emissions of the Io footprint are optically thin and are consequently subject to limb brightening. The studies of the Io footprint brightness usually accounted for this effect assuming that the footprint is an extended structure in the horizontal plane [Clarke et al., 1998; Gérard et al., 2006; Serio and Clarke, 2008]. The present results demonstrate that this hypothesis is arguable and that more complex methods should be used to account for the orientation of the footprint on the images.

[30] Acknowledgments. The author thanks J.-C. Gérard and D. Grodent for their constructive comments. This study was supported by the PRODEX program managed by ESA in collaboration with the Belgian Federal Science Policy Office. This research is based on observations made with the Hubble Space Telescope obtained at the Space Telescope Science Institute, which is operated by AURA Inc.

[31] Masaki Fujimoto thanks the reviewers for their assistance in evaluating this paper.

\section{References}

Bonfond, B. (2009), Morphology and dynamics of the Io UV footprint, Ph.D. thesis, Univ. of Liege, Liege, Belgium.

Bonfond, B., J.-C. Gérard, D. Grodent, and J. Saur (2007), Ultraviolet Io footprint short timescale dynamics, Geophys. Res. Lett., 34, L06201, doi:10.1029/2006GL028765.

Bonfond, B., D. Grodent, J.-C. Gérard, A. Radioti, J. Saur, and S. Jacobsen (2008), UV Io footprint leading spot: A key feature for understanding the UV Io footprint multiplicity?, Geophys. Res. Lett., 35, L05107, doi:10.1029/2007GL032418.

Bonfond, B., D. Grodent, J.-C. Gérard, A. Radioti, V. Dols, P. A. Delamere, and J. T. Clarke (2009), The Io UV footprint: Location, interspot distances and tail vertical extent, J. Geophys. Res., 114, A07224, doi:10.1029/ 2009JA014312.

Chust, T., A. Roux, W. S. Kurth, D. A. Gurnett, M. G. Kivelson, and K. K. Khurana (2005), Are Io's Alfvén wings filamented? Galileo observations, Planet. Space Sci., 53, 395-412, doi:10.1016/j.pss.2004.09.021.
Clarke, J. T., et al. (1996), Far-ultraviolet imaging of Jupiter's aurora and the Io "footprint," Science, 274, 404-409.

Clarke, J. T., et al. (1998), Hubble Space Telescope imaging of Jupiter's UV aurora during the Galileo orbiter mission, J. Geophys. Res., 103 (E9), 20,217-20,236, doi:10.1029/98JE01130.

Clarke, J. T., et al. (2002), Ultraviolet emissions from the magnetic footprints of Io, Ganymede and Europa on Jupiter, Nature, 415(6875), $997-1000$

Connerney, J. E. P., R. Baron, T. Satoh, and T. Owen (1993), Images of excited $\mathrm{H}_{3}^{+}$at the foot of the Io flux tube in Jupiter's atmosphere, Science, 262, 1035-1038.

Connerney, J. E. P., M. H. Acuña, N. F. Ness, and T. Satoh (1998), New models of Jupiter's magnetic field constrained by the Io flux tube footprint, J. Geophys. Res., 103(A6), 11,929-11,940, doi:10.1029/ 97JA03726.

Dols, V., P. A. Delamere, and F. Bagenal (2008), A multispecies chemistry model of Io's local interaction with the plasma torus, J. Geophys. Res., 113, A09208, doi:10.1029/2007JA012805.

Gérard, J.-C., J. Gustin, D. Grodent, P. Delamere, and J. T. Clarke (2002), Excitation of the FUV Io tail on Jupiter: Characterization of the electron precipitation, J. Geophys. Res., 107(A11), 1394, doi:10.1029/ 2002JA009410.

Gérard, J.-C., A. Saglam, D. Grodent, and J. T. Clarke (2006), Morphology of the ultraviolet Io footprint emission and its control by Io's location, J. Geophys. Res., 111, A04202, doi:10.1029/2005JA011327.

Gladstone, G. R., et al. (2007), Jupiter's nightside airglow and aurora, Science, 318, 229-231, doi:10.1126/science.1147613.

Grodent, D., J. Waite, and J.-C. Gérard (2001), A self-consistent model of the Jovian auroral thermal structure, J. Geophys. Res., 106(A7), 12,93312,952, doi:10.1029/2000JA900129.

Grodent, D., B. Bonfond, J.-C. Gérard, A. Radioti, J. Gustin, J. T. Clarke, J. Nichols, and J. E. P. Connerney (2008), Auroral evidence of a localized magnetic anomaly in Jupiter's northern hemisphere, J. Geophys. Res., 113, A09201, doi:10.1029/2008JA013185.

Hess, S., P. Delamere, V. Dols, B. Bonfond, and D. Swift (2010), Power transmission and particle acceleration along the Io flux tube, J. Geophys. Res., 115, A06205, doi:10.1029/2009JA014928.

Hinson, D. P., A. J. Kliore, F. M. Flasar, J. D. Twicken, P. J. Schinder, and R. G. Herrera (1998), Galileo radio occultation measurements of Io's ionosphere and plasma wake, J. Geophys. Res., 103(A12), 29,343-29,358, doi:10.1029/98JA02659.

Jacobsen, S., F. M. Neubauer, J. Saur, and N. Schilling (2007), Io's nonlinear MHD-wave field in the heterogeneous Jovian magnetosphere, Geophys. Res. Lett., 34, L10202, doi:10.1029/2006GL029187.

Jacobsen, S., J. Saur, F. M. Neubauer, B. Bonfond, J. C. Gérard, and D. Grodent (2010), Location and spatial shape of electron beams in Io's wake, J. Geophys. Res., 115, A04205, doi:10.1029/2009JA014753.

Jones, S. T., and Y.-J. Su (2008), Role of dispersive Alfvén waves in generating parallel electric fields along the Io-Jupiter fluxtube, J. Geophys. Res., 113, A12205, doi:10.1029/2008JA013512.

Kivelson, M. G., F. Bagenal, W. S. Kurth, F. M. Neubauer, C. Paranicas, and J. Saur (2004), Magnetospheric interactions with satellites, in Jupiter: The Planet, Satellites and Magnetosphere, pp. 513-536, Cambridge Univ. Press, New York.

Neubauer, F. M. (1980), Nonlinear standing Alfven wave current system at Io: Theory, J. Geophys. Res., 85(A3), 1171-1178, doi:10.1029/ JA085iA03p01171.

Prangé, R., D. Rego, D. Southwood, P. Zarka, S. Miller, and W. Ip (1996), Rapid energy dissipation and variability of the Io-Jupiter electrodynamic circuit, Nature, 379, 323-325, doi:10.1038/379323a0.

Prangé, R., D. Rego, L. Pallier, J. Connerney, P. Zarka, and J. Queinnec (1998), Detailed study of FUV Jovian auroral features with the postCOSTAR HST faint object camera, J. Geophys. Res., 103(E9), 20,195-20,216, doi:10.1029/98JE01128

Saur, J., F. M. Neubauer, J. E. P. Connerney, P. Zarka, and M. G. Kivelson (2004), Plasma interaction of Io with its plasma torus, in Jupiter: The Planet, Satellites and Magnetosphere, pp. 537-560, Cambridge Univ. Press, New York.

Serio, A. W., and J. T. Clarke (2008), The variation of Io's auroral footprint brightness with the location of Io in the plasma torus, Icarus, 197, 368374, doi:10.1016/j.icarus.2008.03.026.

Vasavada, A. R., et al. (1999), Jupiter's visible aurora and Io footprint, J. Geophys. Res., 104(E11), 27,133-27,142, doi:10.1029/1999JE001055. Williams, D. J., and R. M. Thorne (2003), Energetic particles over Io's polar caps, J. Geophys. Res., 108(A11), 1397, doi:10.1029/2003JA009980.

B. Bonfond, Laboratoire de Physique Atmosphérique et Planétaire, Université de Liège, Allée du 6 Août 17, B-4000 Liège, Belgium. (b.bonfond@ulg.ac.be) 